\title{
Selective perception without confounding contributions of decision and memory
}

\author{
STEPHEN J. LUPKER \\ University of Western Ontario, London, Ontario N6A 5C2, Canada \\ and \\ DOMINIC W. MASSARO \\ University of Wisconsin, Madison, Wisconsin 53706
}

\begin{abstract}
Previous research has demonstrated that decision processes and short-term memory limitations contribute to the observed limitation in the span of apprehension in tachistoscopic experiments. The present study addresses the question of whether perceptual factors also contribute to this limitation. Observers were asked to indicate which of four target letters occurred in a four-item test display. The irrelevant background items were either highly confusable or completely nonconfusable with the target letters. The target detection task is designed to bypass short-term memory limitations. In order to eliminate differences in decision processing, the location of the target letter was indicated either slightly before or shortly after the display presentation. The indicator was either an arrow cue or a pattern mask. Performance decreased with increases in the delay of the arrow cue only when the background items were confusable. The pattern mask yielded standard masking functions, but performance with the nonconfusable background items improved more rapidly with increases in processing time than did performance with the confusable background items. These results conform to the hypothesis that attention operates at the perceptual stage of processing. The results were accurately described by a quantification of attentional effects in a general information processing model.
\end{abstract}

In recent years, a general metatheoretical view of visual information processing has emerged. This view is based on the idea of successive stages of processing (e.g., Broadbent, 1958; Massaro, 1975; Sperling, 1960; Sternberg, 1969). Upon presentation of a printed stimulus, a perceptual system resolves figure-ground information from a preperceptual visual representation of the display. The perceptual process takes time and, prior to its completion, may be interfered with by the presentation of a masking stimulus. The figure-ground information that has been established is encoded in terms of abstract character names for each stimulus item. These names are then stored in short-term memory (STM) where they can be rehearsed, recoded, and/or output as responses.

The distinction between a perceptual stage and a STM stage is most apparent in the seminal work on the limited span of apprehension. Sperling (1960)

This research was supported in part by Public Health Service Grant MH-19399. The authors wish to thank Dr. John Theios for allowing us to use his laboratory and equipment while carrying out this research. Requests for reprints may be sent to Stephen J. Lupker, Department of Psychology, University of Western Ontario, London, Ontario N6A 5C2, Canada, or to Dominic W. Massaro, Department of Psychology, University of Wisconsin, Madison, Wisconsin 53706. displayed character arrays of various sizes and configurations for brief periods of time and asked observers to report as many of the characters as possible (the classic whole report procedure). The subjects could maximally report only about four or five characters from the display. However, Sperling (1960), as well as Averbach and Coriell (1961), demonstrated that this limitation could be effectively circumvented by cueing observers to selectively report only certain locations in the display. Sperling's observers were auditorially cued to report only one row of items in the visual display. Performance was very good when the cue followed the display immediately and decreased with cue delay, asymptoting at a level of performance that could be predicted from the whole-report procedure. Averbach and Coriell presented a 2 by 8 array of letters and used a bar marker to cue one letter for report. They found that the probability of report for the cued location was nearly perfect with immediate cues and decreased monotonically with cue delays up to $250 \mathrm{msec}$.

The idea of successive stages makes viable two alternative interpretations of these results. The first locates the limitation in the span of apprehension at the STM stage rather than at the perceptual stage. Since Sperling's subjects performed almost per- 
fectly when the cue followed a nine-item display immediately, all letters must have been identified and stored in STM. The decrease in performance with increasing cue delay could only have resulted from forgetting during the rehearsal and reporting of the information from STM.

The second interpretation of these results does not deny a STM limitation, but also assumes that the span of apprehension is constrained by the perceptual stage of processing. That is, the perceptual stage is limited in the number of characters that it can simultaneously resolve, and the cue allows selective perception of some of the items in the display. According to this view, the items in the tachistoscopic display were not yet perceived at the time the stimulus display was turned off, but information about them was held in a short-duration preperceptual visual storage. The cue allowed the subject to selectively perceive the relevant items before this informaton decayed. Enhanced performance with shorter delays could occur since the sooner the items are cued, the more likely it is that they can be perceived before they have decayed from preperceptual visual storage. Without the cue, the subjects probably distributed their attention among all of the items in the display, making it less likely that the relevant items were perceived.

As a means of testing between these two views, Estes and Taylor $(1964,1966)$ devised a paradigm that reduces the contribution of forgetting from STM. Presented with a brief display of letters containing one of two targets (i.e., a $\mathrm{T}$ or an F), the subject's task was to report which of these targets was present. Given that the other items in the display did not have to be recalled, memory demands did not increase with increases in display size. Even so, performance decreased with increases in the number of items in the display. If STM differences cannot account for the decrease in performance, these results would seem to support the idea of a capacity limitation in the perceptual span of apprehension.

However, Estes and Taylor's results are open to an alternative interpretation, which also has implications for the earlier work on the span of apprehension. Expanding on an idea first proposed by Eriksen and Spencer (1969), Gardner (1973) argued that previous results, such as those of Estes and Taylor, could be explained solely in terms of differences during a decision stage that was unique to the task. Although the subject monitors all of the items in the display, only a single response (i.e., a $\mathrm{T}$ or an $\mathrm{F}$ ) is required. Gardner assumed that each item in the display was processed by a separate perceptual channel, and that the perceptual processing of any item was independent of the number or nature of other items in the display (assuming there are no lateral masking effects). After perceptual processing has gone to completion, the decision process evaluates the estimates of the identities of each of the items and determines the most appropriate response for that display. By increasing the number of items in the display, the number of items that must be evaluated also increases. Therefore, increasing the number of items also increases the probability of mistakenly identifying one of the nontargets as the target. For example, on a given trial in the T-F task, a nontarget $\mathrm{E}$ might look more like an $\mathrm{F}$ than the actual target $T$ looked like a $T$. Increasing the number of nontargets in the display would increase the likelihood of one of these misidentifications, even though the target was seen equally well under all conditions. It follows that the drop-off in performance with increases in the number of nontarget items could be explained in terms of the decision stage without requiring the span of apprehension to be limited by the perceptual stage.

Elaborating on Estes and Taylor's basic paradigm, Gardner independently varied the observer's perceptual and decision processing. In the baseline condition, a single $\mathrm{T}$ or $\mathrm{F}$ character was displayed at one of the four corners of an imaginary square centered around a fixation point. In a second condition, the demands on the perceptual system, but not the demands on the decision system, were increased by filling the remaining three corners of the square with Os (the nonconfusable-background condition). Since the Os and the targets were so dissimilar, Gardner reasoned that the decision stage would not have to consider these items in choosing a response. Therefore, as in the baseline condition, the subject would be able to make a response based solely on the estimate of the identity obtained from the target letter. If this assumption is correct, any difference between the two conditions could be attributed to the increased perceptual load in the nonconfusable-background condition.

In the third condition, the confusable-background condition, the remaining three corners of the square were filled with T-F hybrids (i.e., the symbol F). According to Gardner's assumption of independent perceptual channels, these displays should make the same demands on the perceptual system as those in the nonconfusable-background condition. Given the confusability of the hybrids with the targets, however, the decision stage could not limit its processing to just the target location and would have to make a decision based on all four character locations in the display. This increase in the demands on the decision stage should cause a decrease in performance relative to the nonconfusablebackground condition, because the decision stage could easily mistake one of the hybrids for a target and respond on the basis of this irrelevant infor- 
mation. Supporting Gardner's initial theorizing, there was no difference in performance between the target-alone condition and the nonconfusablebackground condition, but there was a dramatic decrement of $43 \%$ in the confusable background condition relative to these two conditions.

Although differences at the decision stage may be sufficient to describe Gardner's results, the possibility of perceptual selection in this task cannot be ruled out. Given the appropriate cue, perceptual processing could be allocated to a particular location in space, allowing the observer to selectively perceive the contents of that location. The configuration of Os may serve as just such a cue, while the configuration of hybrids would not. Therefore, the target may have received enhanced perceptual processing, in addition to the more optimal decision processing, in the nonconfusable-background condition relative to the confusable-background condition. Gardner (1973) acknowledged this possibility, but argued that differences at the decision stage were sufficient to account for the results. However, although Gardner (1973) described two possible formalizations of the decision stage, a quantitative description of the results was not presented. It is important to know whether the decision stage could account for the extremely large decrease of $43 \%$ when just three confusable hybrids were added to the display. Using Gardner's formalizations, we have been able to account for only about two-thirds of the performance decrement. Therefore, both the possibility of perceptual selection in the task and the possible insufficiency of the decision stage preclude an unambiguous interpretation of the results in Gardner's study.

Previous work has not unambiguously determined whether the perceptual stage is at all responsible for the span of apprehension. In order to test the idea of a perceptual span of apprehension (i.e., limited capacity and selective attention at the perceptual stage of processing), both decision processing and STM will have to be accounted for. The purpose of the present experiment is to provide a strong test of the two alternative hypotheses about the perceptual stage by applying the appropriate controls to the other stages. According to the hypothesis viewing perception as an unlimited capacity stage, the perceptual information gained about the target must be equivalent for each of Gardner's test displays. Differences in the decision stage are entirely responsible for any performance differences. Following this reasoning, if a report cue were presented during perceptual processing, the decision stage could ignore the nontargets and any response could be based solely on the perceptual information obtained from the cued location. Since there is no reason to believe that the report cue will be completely effective, the present experiment also allows an assessment of its effectiveness at the decision stage of processing. According to Gardner's idea of independent parallel channels, the same perceptual information about the target must be available in all conditions. If the report cue is effective, performance should be the same in all conditions.

In order to test whether results such as these would obtain, we used the same basic paradigm and displays as Gardner's. A target letter was presented in a background of nonconfusable nontargets or a background of confusable nontargets. In addition, on most trials a cue indicated the location of the target to be reported. Two kinds of cues were used, a small arrow pointing to the target from the center of the imaginary square, and a small mask covering only the location of the target. Six different stimulus onset asynchronies (SOAs) between the display and cue were used. This allowed us to trace out both cueing and masking functions in both background conditions.

The idealized predictions of the hypothesis of an unlimited-capacity perceptual stage are straightforward. If the arrow is presented during or immediately after perceptual processing, the decision system should be able to ignore the nontargets and, therefore, performance should be independent of both SOA and background condition. Given that a mask disrupts perceptual processing, performance on the masking trials will improve with increases in SOA. Since selective perception cannot occur, however, the same information about the target must be available in both the confusable-background and nonconfusable-background conditions at all points during perceptual processing. Identical masking functions should then be found in the two background conditions, because the mask also acts a cue to allow the subject to respond solely on the basis of the perceptual information derived from the target letter.

Alternatively, according to the hypothesis viewing perception as a limited-capacity stage, the nonconfusable nontargets and the arrow cue may allow selective attention during perception. If so, the idealized results should be as follows. With the nonconfusable background, the arrow will be redundant because the attention switching cue is contained in the display. On these trials, performance should be independent of SOA. Presented with the confusable background, the observer must perceptually process four items until the arrow arrives and allows attention switching. At short SOAs, performance should approach that in the nonconfusable-background condition, since attention can be switched early in perceptual processing. Performance will decrease monotonically with increases in cue delay, however, since perceptual processing will have to be divided among 
four items until the cue is presented and attention is switched. When the mask is used as a location cue, standard masking functions would be found in both background conditions. In the nonconfusablebackground condition, however, the display contains an attention-switching cue, and observers should generally be able to switch their perceptual attention to the target before the mask terminates processing. Because of this period of enhanced perceptual processing of the target, the masking function in the nonconfusable-background condition would be expected to rise faster than the masking function in the confusable-background condition.

\section{METHOD}

\section{Subjects}

Eight University of Wisconsin undergraduates, one male and seven females, received course credit for participating in four 1-h sessions on 4 consecutive days. Two pilot subjects were tested to determine the proper display parameters.

\begin{abstract}
Apparatus
All experimental events were controlled and recorded by a small real-time computer (Digital Equipment Corporation PDP-8). A Tektronix (Type 503 RM) oscilloscope (P15 phosphor) was used to display the characters and was positioned $1.54 \mathrm{~m}$ away from the subject. A chinrest was used to reduce variability in the subject's head position. The P15 phosphor decays to $10 \%$ of its original intensity within $2.8 \mathrm{msec}$. The target characters, the background items, and the mask subtended visual angles of $.2^{\circ}$ in width and $.3^{\circ}$ in height, with $1.7^{\circ}$ visual angle between the characters, the same specifications used by Gardner (1973). The arrow subtended a visual angle of $.4^{\circ}$. On the table in front of the subject was a box with four buttons. The subject was able to initiate a trial by pressing any of these buttons. The subject responded by hitting the appropriate button.
\end{abstract}

\section{Procedure}

On any trial, the subject first fixated on the dot in the middle of the screen and then pushed any button to initiate the display. The four characters were displayed at the four corners of an imaginary square. Each of the characters was displayed within a 5 by 7 dot matrix. Each dot in the display was painted only once such that the total duration of the display was $10 \mathrm{msec}$. One of the characters was the target $(E, F, I$, or $T)$, while the other three characters were noise letters (zeros or hybrids). Only one type of noise letter was used on any trial. The indicator (either an arrow pointing at the target from the center of the square or a mask presented at the same location as the target) lasted $200 \mathrm{msec}$. The mask in this experiment was a rectangle with a cross inside of it such that the lines of all the targets were embedded in the set of lines making up the mask. Six SOAs were used, two of which involved precues $(-25$ and $-75 \mathrm{msec}$ ). For the precue trials, the indicator was not presented during the test display; however, it came back on immediately after the display for the remainder of the $200 \mathrm{msec}$. The other four SOAs were $35,85,160$, and $235 \mathrm{msec}$.

A complete factorial design of target type, target position, background items, indicator, and SOA was employed giving a total of 384 unique trials ( 4 targets by 4 positions by 2 background items by 2 indicators by 6 SOAs). Also, 32 no-cue trials, 16 with the hybrid background and 16 with the zero background, were randomly interspersed among the cued trials. Thus, each trial block contained 416 trials. Each subject was tested on the set of 416 trials once on Day 1 and twice on Days 2, 3, and 4.
The trials for each block were presented in a completely random and unique order. The subjects were not informed of the nature of the upcoming trial, and no response feedback was given. Day 1 was considered practice and the data were disregarded, although the subjects were not informed of this. An attempt was made to keep the overall performance of each subject between $60 \%$ and $65 \%$ correct, on each day. To this end, both the intensity of the display and the amount of illumination in the room were adjusted accordingly, prior to each day.

\section{RESULTS}

The data conform to the predictions derived from the hypothesis that selective attention operates at the perceptual stage. Figure 1 plots performance in ' ${ }^{\prime}$ values for the cueing and masking conditions as a function of SOA and background. The points represent the observed values, and the lines are predictions of a model to be developed in the Discussion section. The $d^{\prime}$ values were computed from the percentage correct scores averaged over all subjects, using the tables for four independent alternatives given by Elliot (1964). Performance in the cueing condition with nonconfusable-background items (open squares) was relatively independent of SOA. Performance in the cueing condition with the confusable background (open circles) was equivalent to that in the nonconfusable background condition at negative SOAs, but decreased with increases in cue delay. The two masking conditions yielded standard masking functions with performance in the nonconfusablebackground condition (closed squares) rising much more rapidly than performance in the confusablebackground condition (closed circles).

The results of the no-cue trials are also shown in Figure 1 on the far right side of the figure. Performance with the nonconfusable background was unaffected by the presence or absence of a cue. In the confusable-background condition, however,

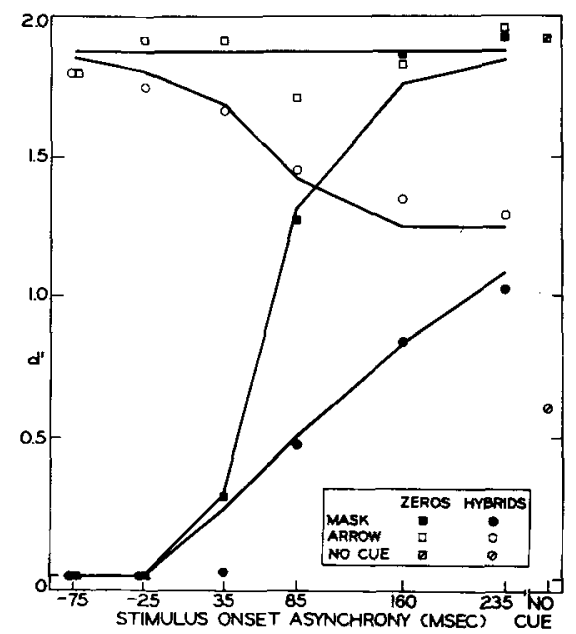

Figure 1. Performance in $d^{\prime}$ values for the cueing and masking conditions as a function of SOA and background conditions. 
there was a substantial decrement in performance when no cue was presented, relative to asymptotic performance at long cue delays.

An analysis was carried out on the percentage correct scores with subject, SOA, indicator, background condition, target type, target location, and days as factors. As can be seen in Figure 1, the Background by SOA by Indicator interaction was significant $[F(5,35)=9.23, p<.001]$. Also significant were the main effects of these variables, as well as their pairwise interactions (all ps $<.001$ ). No other effects reached significance (all ps $>.05$ ).

\section{DISCUSSION}

The results of the present experiment are quite clear-cut. The two masking functions separate as early as $35 \mathrm{msec}$ and asymptote at quite different levels, as predicted by the perceptual selective attention hypothesis. This indicates that perceptual processing must have resolved more of the target in the nonconfusable-background condition than in the confusable-background condition at each of these points. This could only happen, of course, if the observers were somehow applying more perceptual capacity (i.e., selectively attending) to the target in the nonconfusable-background condition relative to the confusable-background condition.

At the same time, the divergent masking functions provide strong evidence against the opposing hypothesis of an unlimited capacity perceptual stage. Since masks terminate, or at least severely attenuate, perceptual processing, their presentation forces the perceptual system to turn over incomplete information to the decision stage. Therefore, masking functions can be used as direct indices of the amount of perceptual processing accomplished. If the perceptual stage were unlimited in capacity, the amount of information about the target should have been equivalent in the confusable-background and nonconfusablebackground conditions at every point during perceptual processing. Thus, when the target is masked, the decision system should receive the same incomplete perceptual information in both background conditions. Since the mask also acts as a report cue, the subsequent decision stage should also have been equivalent in the two background conditions. It follows that the masking functions in the two conditions should also have been equivalent; the results contradict this prediction of an unlimited capacity perceptual stage.

The positive results of selective attention at the perceptual stage do not preclude differences in performance due to the decision stage. Given a confusable background, performance at an SOA of $235 \mathrm{msec}$ is substantially above performance on the no-cue trials. Since perceptual processing should have ended by the time these functions reached asymptote, this difference cannot be due to perceptual selection. With no cue and a confusable background, the subject would be more likely to report one of the background items as the target. Therefore, the decrement in performance in this no-cue condition relative to the asymptotic cueing condition with a confusable background reflects differences in decision processing.

Replicating Gardner's (1973) results, there was a large difference between the confusable-background and nonconfusable-background conditions when no cue was given. The fact that the cue did not facilitate performance with a nonconfusable background indicates that the attention-directing cue in the nonconfusable-background condition is entirely contained in the display. Therefore, decision processing was equivalent for the cue and no-cue condition with a nonconfusable background.

The difference between asymptotic performance with a cue in the confusable-background condition and performance in the nonconfusable-background condition when no cue was given can be attributed to perceptual factors. This difference is reflected by the drop-off in the confusable-background arrowcueing function with increases in SOA. When the cue arrives very early, perceptual attention can be directed to the target. However, the longer the cue is delayed, the more time the observer must process all of the items in the display and the less time available for selectively processing the target. At asymptote, perceptual selection is no longer possible.

Given the contributions of both perceptual and decision processing, it would be valuable to measure the relative contribution of each. Precise empirical measures are not available, however, because the confusable-background functions have not quite reached asymptote at $235 \mathrm{msec}$. Utilizing the description of a selective perception model (developed in the next section), it appears that the contribution of perceptual selection was about twice the contribution of decision selection.

The present experiment was designed to allow a direct assessment of selective perception without confounding contributions of decision and memory. It is important to consider whether the design was successful by evaluating alternative explanations based on decision and memory. One possibile argument is that the report cues are not completely effective and that confusable nontargets in the display still contribute to the final decision. However, performance with an arrow cue at the -75 -msec SOA with a confusable background was equivalent to asymptotic performance in the nonconfusablebackground condition. This means that the arrow cue was as effective as the nonconfusable background, in terms of allowing the decision process to 
make a response based solely on the perceptual information from the target item. It might also be argued that the pattern mask is not an effective report cue. If subects could not use the mask as a report cue, the differences between the two masking functions could be accounted for by the decision process. Two aspects of the data are inconsistent with this idea. First, in the confusable-background condition, asymptotic performance with the pattern mask is much higher than performance when no cue is presented. Second, performance levels with the two types of cues converged at long SOAs. Thus, the pattern mask and the arrow appear to be equally good report cues and as good as the nonconfusable background display.

A second argument might be that forgetting of acquired perceptual information from STM would account for the overall poorer performance in the confusable- than in the nonconfusable-background condition. With a confusable background, a subject will not be certain which item is the target until the location is indicated by the arrow or the mask. It follows that the perceptual information must be preserved either until decisions can be made about all four items or until the indicator. is presented. Any forgetting of this information due to STM limitations would produce poorer performance with the confusable-background than with the corresponding nonconfusable-background condition. Although this explanation could account for the decrease in performance with increases in SOA with an arrow cue, it cannot account for the simultaneous increase in performance with the pattern mask (compare the arrow-and-hybrids and mask-andhybrids functions in Figure 1). In order for a loss of perceptual information to occur, this information must have already been perceived and stored in STM. If this were so, the two kinds of report cues should be equally facilitative. That is, the perceptual resolution of the items in the display would have produced relatively abstract codings of item identity at each position and the cue would simply allow report of the appropriate location. The fact that the mask interferes with performance at short SOAs can only mean that the perceptual resolution of the items was not yet complete. Observing that an arrow cue facilitates performance at the same SOA that the mask interferes with performance is good evidence that the arrow cue is having its facilitative effect at the perceptual stage of processing and not only at the level of STM.

\section{Selective Perception Model}

The results can be described within a general information-processing model (Massaro, 1975). A visual display is transduced by the light receptors, and visual features are detected and placed in a pre- perceptual visual storage (PVS). Perception of a character in the display requires the operation of the primary recognition process. This operation integrates the featural representation in PVS into a synthesized percept which is placed in synthesized visual memory. The primary recognition process takes time, and its contribution follows a negatively accelerating function of processing time. If $d^{\prime}$ represents the degree of resolution of the test item by the primary recognition process, its time course can be described by

$$
\mathrm{d}^{\prime}=\alpha\left(1-\mathrm{e}^{-\theta t}\right)
$$

where $\alpha$ represents the asymptotic resolution given unlimited processing time, $\theta$ represents the rate of processing to this asymptote, $t$ is the processing time available, and $\mathrm{e}$ is the natural logarithm.

The primary support for this description of the perceptual process comes from backward-recognition masking experiments (Massaro, 1975). A short test display is followed, after a variable blank interval, by a masking display. The prototypical finding is that recognition of the test display improves with increases in the blank interval between the test and masking displays. These results indicate that perception was not complete at the offset of the test display; if it were, performance should have been asymptotic at all masking intervals. Furthermore, the unprocessed featural information must have been held in some form of storage in order for perception to continue after the display was turned off. In terms of the present model, this storage would correspond to PVS. Finally, the masking stimulus was effective in terminating the perceptual process, supposedly by interfering with the information held in PVS. The backward-recognition masking results provide concomitant support for each of these conclusions.

The value of $\alpha$ represents the signal-to-noise ratio or the quality of the visual features in preperceptual visual storage. Thus, given unlimited processing time, $\alpha$ is the asymptotic resolution level of the target. In the present model, feature detection can not be modified by selective attention and, therefore, $\alpha$ is independent of the background condition, the nature of the cue, and the cueing interval. The value of $\Theta$ can be thought of as the proportion of the features in PVS that are resolved and integrated per unit of time by the primary recognition process. Selective attention in the current model can be localized at $\Theta$, the rate of perceptual processing of the features in PVS. Subjects should be able to process an item at a faster rate, to the extent that the item can be selectively processed. The value of $\Theta$, indexing the rate of target processing, should be larger when attention is devoted to that item relative to the case when attention must be divided among 
four items. If processing time were unlimited, however, selective attention performance would not be better than divided attention, since any deficit caused by the slower processing rate in divided attention could be compensated for by additional processing time.

Performance in a given task with unlimited processing time will not necessarily reach $\alpha$, however, since PVS imposes a limitation on the effective processing time available. Given a short test-display presentation, the rate of processing may not be large enough to insure that processing has been completed before the information in PVS has decayed. It follows that asymptotic performance with a short display and large processing time should be lower than asymptotic performance in a comparable condition in which the display remains on during the processing interval. This result has been obtained in both visual and auditory tasks (Eriksen \& Eriksen, 1971; Massaro, 1972). The general form of Equation 1 must, therefore, account for the duration of PVS. If $t_{D}$ equals the duration of PVS, Equation 1 will hold for values of $t$ less than $t_{D}$, whereas $t_{D}$ will be substituted for $t$ for values of $t$ greater than or equal to $t_{D}$. Description of backward recognition masking results requires estimation of $\alpha, \theta$, and $t_{D}$.

In the current task, backward-recognition masking occurred in both the confusable-background and nonconfusable-background masking conditions. If the nonconfusable background allows switching of attention to the target letter, then the rate of processing the target letter should be larger after attention has been switched. That is, the rate of selectively processing one item should be larger than when attention is divided among four items. Incorporating the concept of switching time and differential rates of processing in Equation 1 gives the following predictions for the backward-masking functions in the two background conditions. For the confusable background where switching attention cannot occur,

$$
\begin{array}{ll}
d^{\prime}=\alpha\left(1-e^{-\Theta_{4} t_{s}}\right) & \text { for } t_{s}<t_{D} \\
d^{\prime}=\alpha\left(1-e^{-\Theta_{4} t_{D}}\right) & \text { for } t_{s} \geqslant t_{D},
\end{array}
$$

where $t_{s}$ is equal to the SOA and $\Theta_{4}$ is equal to the rate of processing the target item when attention must be divided among all four items in the test display. In the case of the nonconfusable background, it is assumed that attention switching can occur. Therefore,

$$
\begin{array}{r}
\mathrm{d}^{\prime}=\alpha\left(1-\mathrm{e}^{-\Theta_{4} t_{s o}-\Theta_{1}\left(t_{s}-t_{s o}\right)}\right) \\
\quad \text { for } t_{s o}<t_{s} \text { and } t_{s}<t_{D}
\end{array}
$$

$$
\begin{aligned}
& \mathrm{d}^{\prime}=\alpha\left(1-\mathrm{e}^{-\Theta_{4} \mathrm{t}_{\mathrm{so}}-\Theta_{1}\left(\mathrm{t}_{\mathrm{D}}-\mathrm{t}_{\mathrm{so}}\right)}\right) \\
& \quad \text { for } \mathrm{t}_{\mathrm{s}} \geqslant \mathrm{t}_{\mathrm{D}} \geqslant \mathrm{t}_{\mathrm{so}} \\
& \mathrm{d}^{\prime}=\alpha\left(1-\mathrm{e}^{-\Theta_{4} \mathrm{t}_{\mathrm{s}}}\right) \\
& \quad \text { for } \mathrm{t}_{\mathrm{so}} \geqslant \mathrm{t}_{\mathrm{s}} \text { and } \mathrm{t}_{\mathrm{s}}<\mathrm{t}_{\mathrm{D}} \\
& \mathrm{d}^{\prime}=\alpha\left(1-\mathrm{e}^{\left.-\Theta_{4} \mathrm{t}_{\mathrm{D}}\right)} \quad \text { for } \mathrm{t}_{\mathrm{so}} \geqslant \mathrm{t}_{\mathrm{D}} \text { and } \mathrm{t}_{\mathrm{s}} \geqslant \mathrm{t}_{\mathrm{D}},\right.
\end{aligned}
$$

where $t_{\text {so }}$ is the time required to switch attention to the target with the nonconfusable-background displays and $\theta_{1}$ is the rate of processing the target item when attention is focused on that item. Equations 4 and 5 show that the test item is processed at a rate of $\Theta_{4}$ during the switching time and then is processed at a rate of $\theta_{1}$ after switching occurred. Switching time involves the time to localize the indicator and the time to dedicate processing to the appropriate item. In terms of the nonconfusable background, the location with straightline features will be the relevant location to be processed. With confusable hybrids in the display, all four items will have straight-line features and no switching of attention can occur.

In the backward-masking conditions, total processing time, $t$, is measured from the onset of the test display to the onset of the masking stimulus, unless $t_{s}$ (the SOA) is greater than $t_{D}$ (the duration of PVS). In the cueing conditions, however, total processing time, $t$, is only limited by the duration of PVS, $t_{D}$. We assume that the subject processes the display as in the backward-masking condition, but now the cue indicator allows the subject to switch attention (if he has not already done so) and process at the selective attention rate $\theta_{1}$. For the confusable background, performance should be given by

$$
\begin{array}{ll}
d^{\prime}=\alpha\left(1-e^{-\Theta_{A}\left(t_{s}+t_{s c}\right)-\Theta_{1}\left(t_{D}-t_{s}-t_{s c}\right)}\right) & \text { for }\left(t_{s}+t_{s c}\right)<t_{D} \\
d^{\prime}=\alpha\left(1-e^{-\Theta_{4} t_{D}}\right) & \text { for }\left(t_{s}+t_{s c}\right) \geqslant t_{D},
\end{array}
$$

where $t_{s c}$ is the switching time to the arrow cue. The subject processes at a rate $\theta_{4}$ until the cue indicator has been presented and he has switched attention, and then he processes at a rate of $\theta_{1}$ for the remainder of the duration of PVS (Equation 8). If the cue indicator is delayed long enough so that the subject cannot switch attention to the relevant item before PVS has decayed, he processes at a rate $\theta_{4}$ for the duration of PVS, $t_{D}$ (Equation 9). Given that switching time is critically dependent on 
resolving the appropriate cue and the change in processing allocation, it is necessary to estimate a different switching time in the confusable-background cueing. condition than in the nonconfusablebackground conditions. It seems reasonable that the switching time to the arrow in the confusablebackground condition should be significantly longer than that in the nonconfusable-background condition. With the zero background, the cueing arrow is redundant and performance is simply

$$
\begin{array}{ll}
d^{\prime}=\alpha\left(1-e^{-\Theta_{4} t_{s o}-\Theta_{I}\left(t_{D}-t_{s o}\right)}\right) & \text { for } t_{s o}<t_{D} \\
d^{\prime}=\alpha\left(1-e^{-\Theta_{4} t_{D}}\right) & \text { for } t_{s o} \geqslant t_{D},
\end{array}
$$

regardless of the cueing interval. Therefore, performance should be constant in the cueing condition with the nonconfusable background, and independent of the interval between the onset of the display and the onset of the cue indicator.

The above equations give performance in the cases in which the test display precedes the onset of the mask or bar marker. When the cue precedes the test display, switching can begin and the subject will have more time to process the display at the larger rate, $\theta_{1}$. In the confusable-background condition

$$
\begin{array}{ll}
\mathrm{d}^{\prime}=\alpha\left(1-\mathrm{e}^{-\Theta_{4}\left(t_{s c}-t_{p}\right)-\theta_{1}\left(t_{D}-t_{s c}+t_{p}\right)}\right) & \text { for } t_{s c}>t_{p} \\
d^{\prime}=\alpha\left(1-e^{-\theta_{1} t_{D}}\right) & \text { for } t_{p} \geqslant t_{s c}
\end{array}
$$

where $t_{p}$ is the duration between the onset of the precue indicator and the onset of the test display.

The logic is similar for the nonconfusablebackground condition with a precue, except that the precue may be less beneficial if $t_{\mathrm{so}}$, the time required to switch attention when the nonconfusable background is presented, is significantly less than $t_{s c}$, the time required to switch attention to the position indicated by the arrow. If $t_{s c}<\left(t_{p}+t_{s o}\right)$, then the precue can facilitate performance in the nonconfusablebackground condition and performance is given by Equation 12. If $t_{s c} \geqslant\left(t_{p}+t_{s o}\right)$, the precue will not facilitate performance and Equation 10 gives the predicted results.

In order to apply the present model to the current results, it is necessary to estimate the six parameters, $\alpha, \Theta_{4}, \Theta_{1}, t_{s o}, t_{s c}$, and $t_{D}$. The observed $d^{\prime}$ scores were computed by first taking the overall average percentage correct scores for each condition and then using the tables for four alternatives given by Elliot (1964). The predicted d' values were determined by estimating the six parameters to minimize the squared deviations between the predicted and observed values using the iterative subroutine STEPIT (Chandler, 1969). Figure 1 presents the predicted and observed results. The root mean square deviation was .075 . The values of $\alpha$ and $t_{D}$ were estimated to be 1.87 and $299 \mathrm{msec}$, respectively. The switching time with the nonconfusable background was $33 \mathrm{msec}$, whereas switching time for the arrow cue was $196 \mathrm{msec}$. The shorter switching time with the nonconfusable background is not unreasonable, considering that the crucial information of straight vs. curved lines is contained in the display itself. The estimate of 196-msec switching time for the arrow cue agrees nicely with more direct estimates of switching time to a cue marker (Colegate, Hoffman, \& Eriksen, 1973; Eriksen \& Coliins, 1969). Finally, the $\Theta_{4}$ rate of processing of the target when the other three items must also be processed was estimated to be 3.68 , compared to the estimated $\theta_{1}$ rate of 21.24 when selectively processing the target item. The larger value of $\Theta_{1}$ relative to $\Theta_{4}$ reflects the contribution of selective perception in the model.

\section{Related Results and Models}

Eriksen and his colleagues have also demonstrated the limited capacity nature of visual perception. In the Eriksen and Eriksen (1974) study, a single target was presented above a fixation point and subjects were instructed to classify it by moving a lever in the appropriate direction. Reaction times to classify the target increased significantly when additional letters were placed on each side of the target. The authors placed the flanking letters $.5^{\circ}$ from the target to eliminate lateral interference effects. Flanking letters slowed reaction times even when the flanking letters were identical to the target. Eriksen and Hoffman (1972) asked subjects to name a target letter that was indicated by a bar marker occurring simultaneously with the target. Other letters slowed down the naming response to the extent that they occurred near the target letter in space and time. A small slowing effect was also demonstrated when black disks were placed near the target. These results show that visual processing is limited in capacity, since other items in the display slow down processing of the target item. Selective perception is also demonstrated by the finding that black disks in the display produced less interference than nontarget letters. According to our view, the disks required less processing capacity than the nontarget letters, which allow more capacity to be devoted to processing the target letter. These experiments are consistent with the results of the present study and with the proposed information-processing model.

The notion of an unlimited perceptual stage plays a central role in a recent proposal by Kinchla (1974, 1977). Each stimulus in a visual display is processed 
in an independent manner, so that the encoding of each element depends only on the element from which it originated. The independent codings are then weighted and summed, and this sum is compared to a criterion value to determine the appropriate response. While this model has had success in describing results in similar tasks, it cannot predict the selective perception results observed in the present experiment. Selective attention could be incorporated into Kinchla's model by allowing the encodings of each element to be influenced by the nature of the background items and the arrow cue, and/or permitting the weights to change as a function of background and the arrow cue. It is important to note, however, that either of these changes would require abandoning the central assumption of an unlimited-capacity perceptual stage.

The assumption of an unlimited capacity perceptual stage has also played a central role in a recent proposal of Schneider and Schiffrin (1977). All sensory inputs are first encoded within an automatic processing system, regardless of the nature of the surrounding stimuli. Any differences between the nonconfusable and confusable backgrounds must result from postperceptual processing. Although postperceptual processing usually involves a slow controlled search executed by the decision stage, certain circumstances allow postperceptual processing to be accomplished automatically. The most straightforward explanation of our results within this framework would be that controlled search was necessary with the confusable background but not with the nonconfusable background. However, the masking paradigm used in the present experiment was specifically designed to make this type of controlled search unnecessary in all conditions. When the mask is presented early in perceptual processing, it not only serves to disrupt perceptual processing, but also serves to localize the target. This location cue permits the subject to disregard the nontargets at the decision stage and circumvent the controlled search. (That subjects can ignore the nontargets given a location cue is demonstrated by the equivalent performance in the two cueing functions with confusable and nonconfusable backgrounds at negative SOAs.) Therefore, it does not seem reasonable to assume that controlled search occurred in the confusable-background but not the confusable-background displays at short SOAs. Given the masking-function differences at 35 and $85 \mathrm{msec}$, a modification of Schneider and Shiffrin's framework appears to be necessary.

One remaining possible interpretation of the present results is to attribute the large decrement in performance with the confusable-background items to lateral masking (interference) effects (Estes, 1972; Gardner, 1973). In the interactive-channels model of Estes (1972), for example, detectors of a par- ticular target feature can be inhibited to the extent that the target feature is closely surrounded by similar features. The large spacing between the test items $\left(1.7^{\circ}\right)$ in the present study was enforced to preclude this possibility. To date, no one has established lateral masking between items at this distance.

More recently, Bjork and Murray (1977) found feature-specific inhibition at a distance of about $.75^{\circ}$, and it might be argued that featural interactions can occur at even greater distances. Therefore, it is fortunate that the present experiment has an independent measure of differential lateralinhibition effects for the confusable and nonconfusable displays. Our results provide both empirical and theoretical evidence that such feature interactions did not occur. Empirically, the performance with the confusable-background items was identical to performance with the nonconfusable-background items at a precue interval of -75 -msec SOA. Thus, when selective attention can be optimally directed, no overall performance differences between background conditions were observed. Theoretically, a good description of the data was given by a model which assumes that the potential signal-to-noise ratio of the target item $(\alpha)$ is independent of the confusability of the background items. Such would not be the case if low-level feature interactions between items were occurring in the present experiment. Therefore, it appears that lateral masking did not contribute to the changes in performance as a function of background, backward masking, and cueing.

\section{Conclusion}

The present study attempted to evaluate the role of selective attention at the perceptual processing stage while simultaneously controlling for decision and short-term memory contributions. The results showed that selective attention to a spatial location does occur. Thus, the data were quite adequately described by a quantitative model that assumes that the rate of perceptual processing of a location in space can be influenced by a retroactive cue specifying the appropriate spatial location to attend to.

\section{REFERENCES}

Averbach, E., \& Coriell, A. S. Short-term memory in vision. Bell System Technical Journal, 1961, 40, 309-328.

Bjork, E. L., \& Murray, J. T. On the nature of input channels in visual processing. Psychological Review, 1977, 84, 472-484.

Broadbent, D. E. Perception and communication. New York: Pergamon Press, 1958.

Chandler, J. P. Subroutine STEPIT-Finds local minima of a smooth function of several parameters. Behavioral Science, $1969,14,81-82$.

Colegate, R. L., Hoffman, J. E., \& Eriksen, C. W. Selective encoding from multielement visual displays. Perception \& Psychophysics, 1973, 14, 217-224. 
Elliot, P. B. Tables of d'. In J. A. Swets (Ed.), Signal detection and recognition by human observers. New York: Wiley, 1964.

Eriksen, C. W., \& Collins, J. F. Temporal course of selective attention. Journal of Experimental Psychology, 1969, 80, 254-261.

ERIKSEN, C. W., \& ErIKsen, B. A. Visual perceptual processing rates and backward and forward masking. Journal of Experimental Psychology, 1971, 89, 306-313.

ERIKSEN, B. A., \& ERIKSEN, C. W. Effects of noise letters upon the identification of a target letter in a nonsearch task. Perception \& Psychophysics, 1974, 16, 143-149.

ERIKSEN, C. W., \& Hoffman, J. E. Temporal and spatial characteristics of selective encoding from visual displays. Perception \& Psychophysics, 1972, 12, 201-204.

ERIKSEN, C. W., \& SPENCER, T. Rate of information processing in visual perception. Some results and methodological considerations. Journal of Experimental Psychology Monograph, 1969, 79(2, Part 2).

Estes, W. K. Interactions of signal and background variables in visual processing. Perception \& Psychophysics, 1972, 12, 278-286.

Estes, W. K., \& TAYLOR, H. A. A detection method and probabilistic models for assessing information processing from brief visual displays. Proceedings of the National Academy of Sciences, 1964, 52, 446-454.

Estes, W. K., \& TAYLoR, H. A. Visual detection in relation to display size and redundancy of critical elements. Perception \& Psychophysics, 1966, 1, 9-16.
GARDNER, G. T. Evidence for independent parallel channels in tachistoscopic perception. Cognitive Psychology, 1973, 4, 130-155.

Kinchla, R. A. Detecting target elements in multielement arrays: A confusability model. Perception \& Psychophysics, 1974, 15, 149-158.

KInchla, R. A. The role of structural redundancy in the perception of visual targets. Perception \& Psychophysics, 1977, 22, 19-30.

Massaro, D. W. Stimulus information vs. processing time in auditory pattern recognition. Perception \& Psychophysics, 1972, 12, 50-56.

MASSARO, D. W. Experimental psychology and information processing. Chicago: Rand McNally, 1975.

Schneider, W., \& Shiffrin, R. M. Controlled and automatic human information processing: I. Detection, search and attention. Psychological Review, 1977, 84, 1-66.

SPERLING, G. The information available in brief visual presentations. Psychological Monographs, 1960, 74(11, Whole No. 498).

STERNBERG, $\mathrm{S}$. The discovery of processing stages: Extensions of Donders' method. Acta Psychologica, 1969, 30, 276-315.

(Received for publication July 6, 1978; revision accepted October 24, 1978.)

\section{ERRATUM}

Fagot, R. F. A theory of relative judgment. Perception \& Psychophysics, 1978, 24, 243-252-Change Study No. 4 to Study No. 3, p. 246, column 2, lines 12, 15, and 33; p. 251 , column 1, lines 6 and 7 from bottom. Change Study No. 10 to Study No. 9, p. 246, column 2, line 29. Change Study No. 5 to Study No. 4, p. 249, column 1, caption Figure 2 and line 2. Change Study No. 3 to Study No. 2, p. 249, column 2, line 2, and caption Figure 3. 